\title{
¿Qué pasará con la fecundidad en Brasil?*
}

\section{Ana María Goldani**}

De acuerdo con Naciones Unidas, Brasil se encuentra entre los países con niveles intermedios de fecundidad, es decir, su tasa global de fecundidad está por encima del reemplazo y por debajo de cinco hijos por mujer. Con el fin de recabar información sobre el descenso de la fecundidad en Brasil, la autora entrevistó a destacados demógrafos brasileños durante la conferencia de las Naciones Unidas dedicada a los países con niveles intermedios de fecundidad. Las preguntas que realizó son las siguientes: 1) ¿Seguirán las tasas globales de fecundidad en Brasil las tendencias de los llamados países desarrolados, y alcanzarán niveles por debajo del reemplazo, o se estabilizarán alrededor de los niveles de reemplazo?; 2) en su opinión, ¿cómo se comportará la fecundidad en Brasil en los próximos cincuenta años?; 3) ¿cuál es su estimación de la tasa global de fecundidad para los siguientes periodos: 2000-2005, 2005-2025, 2025-2050?; 4) ipor qué piensa que éste será el promedio de los compontamientos reproductivos esperados en cada uno de los periodos?; 5) en su opinión, ¿̇cuáles son los factores o los principales indicadores que podrian ser particularmente relevantes y útiles para formular supuestos plausibles en relación con el futuro de la fecundidad en Brasil?

Basada en la revisión de literatura y en las respuestas de los demógrafos, la autora examina las proyecciones de continuos descensos de la fecundidad y las fuerzas que probablemente perfilarán las tendencias de la futura fecundidad. A partir de los supuestos de Naciones Unidas para estimar las proyecciones de población de Brasil, discute acerca de las tendencias y los diferenciales socioeconómicos en la fecundidad brasilena, la evolución de sus determinantes próximos y cómo ellos afectan los supuestos de Naciones Unidas en relación con las tendencias futuras de la fecundidad. También presenta los pronósticos de los demógrafos brasileños sobre el futuro de la población de Brasil para las próximas décadas. Finalmente especula sobre las fuerzas que darán forma a la fecundidad de dicho país en el futuro, enfatizando el rol de su legado demográfico y su comportamiento actual, asi como la ley de esterilización y las políticas públicas vigentes, y el papel de las relaciones de género y del apoyo público.

* Artículo presentado en la Conferencia de Naciones Unidas de la Reunión del Grupo de Expertos: "Completando la Transición de la Fecundidad" en Nueva York, del 11 al 14 de marzo de 2002. Agradezco a mis colegas brasileños (los EDB) sus respuestas a mi encuesta y sus comentarios y sugerencias sobre el futuro de la fecundidad en Brasil. No obstante, reconozco mi completa responsabilidad por el contenido de este artículo. También agradezco a Aida V. Lazo su ayuda en el seguimiento de los EDB y el haber conseguido la información, y a Ana Paula P. Walker (UCLA), Ana Roberta Pascom (IPEA) y Luciano Duarte (ENCE) su ayuda en la investigación. Particularmente agradezco a Eduardo Eric Telles sus comentarios y revisiones a este articulo. Traducción de Silvia Luna Santos.

** Profesora visitante en el Departamento de Sociología y Estudios Latinoamericanos de la Universidad de California (UCLA). En Brasil, profesora asociada al Programa de Mestrado en Estudos Populacionais da Escola Nacional de Ciencias Estatisticas (ENCE/IBGE) Río de Janeiro e investigadora del PAGU, Nucleo de Estudos de Genero da Universidade Estadual de Campinas (Unicamp), Campinas, São Paulo. Correo electrónico: anagold isop.ucla.edu 
Palabras clave: fecundidad, proyecciones de población, metodología, género y opinión pública.

Fecha de recepción: 11 de junio de 2002.

Fecha de aceptación: 15 de agosto de 2002.

\section{Contexto}

Pese a la falta de una política oficial de planificación familiar, en la segunda mitad del siglo $\mathrm{xX}$ ocurrieron cambios demográficos sin precedentes en Brasil, marcando el comienzo de una época de tasas globales de fecundidad cercanas al nivel de reemplazo. De acuerdo con los censos demográficos, la población total en Brasil era de 52 millones en 1950 y de 170 millones en el 2000. Esto se tradujo en que la tasa de crecimiento anual promedio de $3 \%$, vigente al inicio del periodo, cayó hasta llegar a $1.5 \%$ anual en los años recientes. La esperanza de vida al nacimiento se incrementó de 44 a 68 años y la tasa global de fecundidad disminuyó de 6.2 a 2.2 en el mismo periodo. Los cambios en la esperanza de vida y en la tasa global de fecundidad fueron avasalladores, ya que se extendieron a todos los estratos sociales, grupos étnicos y regiones, llegando a transformar el curso de vida promedio y la estructura familiar de los brasileños. Aunque los componentes demográficos de tales cambios son bien conocidos, no se cuenta con explicaciones integrales respecto de su acelerado paso.

Varios autores han examinado la multiplicidad de factores que subyacen al rápido descenso de la fecundidad en Brasil, y en su mayoría coinciden en que, además de sus importantes contribuciones, todavía hace falta llegar a una comprensión completa del fenómeno. En la revisión que Martine hace de esos estudios, advierte que hay un consenso respecto a que varios actores institucionales -incluyendo al Estado, la Iglesia católica, los movimientos de mujeres, el sistema de población y los profesionales del sector salud-contribuyeron a la baja de la fecundidad, pero sus influencias no fueron anticipadas ni intencionadas. Este autor también menciona que muchos estudios han priorizado los efectos de la "modernización", y generalmente han confirmado la relación inversa entre la fecundidad, por un lado, y el ingreso y la educación por el otro. Sin embargo no perciben claramente el impacto de la participación de la mujer en el mercado de trabajo. Asimismo, el proceso de urbanización fue otro factor que contribuyó a acelerar el descenso de la fecundidad "porque está aso- 
ciado con una amplia gama de cambios económicos y políticos que transformaron el país durante la última mitad del siglo" (Martine, 1996: 70).

Durante dicho periodo el Estado siguió el modelo de industrialización basado en la sustitución de importaciones característico del régimen de crecimiento de la economía brasileña desde la década de 1950 hasta la de 1970, lo cual suponía un activo rol del Estado -aunque no muy eficiente- en la provisión de bienes básicos y servicios. Con la crisis económica de finales de los setenta y particularmente de principios de los ochenta, casi todos los sistemas de protección social empezaron a mostrar señales de deterioro y hasta de colapso, lo que resultó en una mayor exclusión social. Como consecuencia, el patrón de crecimiento urbano-industrial con exclusión social generó en Brasil esa sociedad de masas y de consumo que describe Faria: las políticas gubernamentales que regulaban el crédito directo al consumo, las telecomunicaciones, los beneficios de la seguridad social y el cuidado de la salud llevaron a una "integración real o simbólica de la población en un mercado de consumo" que cambió el comportamiento individual, para incluir una creciente demanda de regulación de la fecundidad marital (Faria, 1997, 1998: 187).1

Entre los desafios que enfrentan los demógrafos brasileños al analizar el comportamiento reproductivo de su país a lo largo del tiempo se encuentra la gran diversidad regional que ha caracterizado su desarrollo. Hallazgos recientes muestran que existe una fuerte y consistente relación entre la baja de la fecundidad y los notables cambios que se dieron entre 1960 y 1991 en las circunstancias sociales y económicas. Por ejemplo, muestran la influencia de los cambios en la educación de la madre y el impacto de la electrificación de las viviendas en el ritmo del descenso de la fecundidad a lo largo de la transición (Potter et al., 2002). Durante el mismo periodo se incrementaron la escolaridad de la mujer y su participación en el mercado

' Como consecuencia no esperada de estas políticas se presentó una creciente demanda de control de la fecundidad entre las mujeres, que casi pudieron satisfacer el mercado y, en menor medida, las agencias de planificación familiar no gubernamentales. El número de pildoras orales compradas anualmente se incrementó de 1.7 millones de ciclos en 1960 a 61.2 millones en 1980. La falta de servicios de planificación fa miliar gubernamentales gratuitos, el alto costo de las pildoras, su alta tasa de fracaso, y sus efectos colaterales, ocasionaron el desprestigio general de los métodos anticonceptivos reversibles. Aparte de los riesgos del aborto ilegal, esto abrió el camino para que la esterilización se convirtiera en el método de anticoncepción preferido en Brasil (Corrêa et al, 1998). 
laboral, lo cual está íntimamente correlacionado con el descenso de la fecundidad de la generación o cohorte en ese lapso (Lam y Duryea, 1999; Rios Neto, 2000). Asimismo los altos niveles de desigualdad social y la considerable movilidad social característicos de la sociedad brasileña parecen ser factores de peso en las tendencias recientes de la fecundidad (Pastore y Silva, 2000) . ${ }^{2}$ Sin embargo, esos factores parecen haber perdido relevancia para los demógrafos, ${ }^{3}$ dado el extendido y rápido ritmo del descenso de la fecundidad, en gran parte atribuido a la esterilización femenina. Entre las brasileñas casadas la esterilización aumentó de 27 a $40 \%$ entre 1986 y 1996, y únicamente la anticoncepción oral se mantuvo como un método adicional de control natal extensamente utilizado (Benfam, 1997).

Las críticas de las feministas ${ }^{4}$ respecto a que tales explicaciones estructurales no consideraban los altos costos que pagaban las mujeres por la transición de la fecundidad sometiéndose a esterilizaciones, cesáreas y abortos clandestinos, ${ }^{5}$ llevaron a los demógrafos a examinar estas nuevas explicaciones sobre el descenso de la fecundidad. Aunque la esterilización surgió inicialmente entre las clases altas en Brasil, hoy día es común en todos los estratos, generaciones, grupos raciales y otros segmentos de la sociedad, lo que ha resultado en una "cultura de la esterilización" (Berquó, 1999). La prevalencia de parejas de madres e hijas o hermanas que han sido esterilizadas aumenta con el nivel educativo, aunque también se presenta entre mujeres sin educación (Berquó, 1999a: 214).

${ }^{2}$ En 1998 el índice de desarrollo humano (de 0.74 para Brasil) reflejó una serie de indicadores, como una tasa de pobreza de $28 \%$, un ingreso per cápita de alrededor de 250 dólares de EU por mes, e hizo evidente la desigualdad del ingreso, que es la mayor en el mundo entre los principales países.

${ }^{3}$ Casi todos los estudios que se ocupan de los efectos de las diferencias por estrato social y de la rápida proletarización en el comportamiento reproductivo, son de principios de la década de 1980.

" En la década de 1980 las feministas brasileñas ya habían observado el incremento de la demanda para regular la fecundidad entre las mujeres pobres, la precariedad de los servicios de planificación familiar y de salud, así como los efectos del aborto ilegal y de la inequidad de género. Ya para entonces las feministas habían llamado la atención de los demógrafos sobre la necesidad de considerar las experiencias y motivaciones de las mujeres en el análisis de la transición demográfica en Brasil (Corrêa, Piola y Arillha, 1998).

${ }^{5}$ Las estimaciones del número de abortos en Brasil en 1996 varian entre 800000 y 1100000 . Alrededor de 262000 de esas mujeres recibieron atención médica en hospitales públicos (SUS) debido a las complicaciones derivadas del aborto, las cuales representaron la quinta causa de hospitalización obstétrica. Es importante señalar que tales complicaciones representan $9 \%$ de la mortalidad materna (Formiga, 1999: 160). 
Los demógrafos también han concluido que la adopción de nuevas tecnologías anticonceptivas en Brasil no ha sido resultado de elecciones individuales, sino de "procesos colectivos" apoyados fuertemente por la comunidad de médicos, quienes parecen haber desempeñado un papel importante en el proceso de elección (Potter, 1999; Caetano, 2001). Potter informa que en los primeros años del descenso de la fecundidad, cuando la medicina brasileña y la política de salud pública cambiaron de la prevención y control de enfermedades hacia los cuidados curativos especializados en hospitales, las mujeres solicitaban intervenciones quirúrgicas de manera creciente, incluyendo esterilizaciones en hospitales privados afiliados al sistema de seguridad social. Además de su creciente demanda de control natal, se observaba que los médicos y hospitales del sistema público promovían los partos por cesárea, ya que se pagaban al doble de los partos vaginales. ${ }^{6}$ Por consiguiente, varias cesáreas eran innecesarias, y en 1996 llegaron a representar $71 \%$ de las esterilizaciones brasileñas. Mientras en la mayoría de las regiones era más probable que las esterilizaciones se llevaran a cabo durante un parto por cesárea pagado por el paciente, en el Noreste gran parte de las esterilizaciones no requirió la cesárea, y la mayoría de ellas $(70 \%)$, fue pagada por médicos y políti$\cos \left(\right.$ Caetano, 2001). ${ }^{7}$

En consecuencia, una explicación general acerca de la esterilización en Brasil es que es

el resultado de la asociación de una creciente demanda de anticoncepción acompañada de la falta de políticas públicas efectivas y de opciones de control natal pobres, de la influencia de doctores en un medio en

${ }^{6}$ A finales de la década de 1970 , como respuesta al rápido incremento de la tasa de partos por cesárea, la lista gubernamental para el reembolso de partos se ajustó a fin de que se redujera, y eventualmente se eliminara, la prima pagada por las cesáreas, equiparándola con la de los partos vaginales. Sin embargo, fue en 1999 cuando los hospitales afiliados al sistema público recibieron alrededor de 2.4 veces más procedimientos de cesáreas en relación a los partos vaginales (Caetano, 2001).

${ }^{7}$ La Noreste es una de las cinco macrorregiones de Brasil, donde habitaba $43 \%$ de la población brasileña en 1996, y 36\% de ella vivía en zonas rurales. En esta región, considerada como la más pobre del país, es donde existe una mayor desigualdad. Allí, el descenso de la fecundidad se rezagó al menos una década en comparación con las otras cuatro macrorregiones. Mientras que en el Noreste las tasas globales de fecundidad disminuyeron de 6.1 a 3.1 en el periodo 1980-1996, dichas tasas descendieron de 3.6 a 2.3 en el Sur, y de 3.2 a 2.1 en el Sureste, zonas consideradas como las más ricas. En la región Gentro-Oeste la tasa global de fecundidad disminuyó de 4.5 a 2.3 , y en la región Norte de 6.4 a 2.7 (Carvalho,1997-1998). 
que se difundia la medicina curativa en hospitales, y de la capacidad de penetración del comportamiento político caracterizado por la provisión de bienes y servicios a los pobres a cambio de votos (como en el caso específico de la región Noreste) (Caetano, 2001:1).

Es necesario emprender una reconstrucción histórica de los factores que coadyuvaron a los cambios en roles, propósitos, motivos e intenciones sociales, que son cruciales para entender los procesos y causas del cambio en la fecundidad de Brasil. Con este propósito yo sugiero que la fecha de inicio del descenso de la fecundidad no es a mediados de la década de 1960, como con frecuencia se asegura en los estudios sobre Brasil, aunque este fuera un momento decisivo hacia la generalización y la aceleración del proceso de descenso. ${ }^{8}$ Es más, creo que el discurso médico higienista de mediados del siglo XIX, reforzado por el movimiento eugenista de principios del $\mathrm{XX}$, y la revolución anticonceptiva en la década de los sesenta, fueron socialmente asimilados y legitimados, por lo que los brasileños creyeron en los beneficios que podian ofrecer. También sostengo que de esta compleja interacción surgió un interesante conjunto de elementos que apuntan hacia un perfil de política que afecta la reproducción de la población y va más allá de las concepciones neomalthusianas o de las medidas que pretenden controlar el crecimiento demográfico (Goldani, 2001).

Además, en el desarrollo de los arreglos institucionales y las interacciones arriba descritas encontramos indicios importantes de que los sistemas de género, las prácticas del mercado laboral y algunos elementos del sistema administrativo y legal nacional constriñeron el comportamiento reproductivo de las mujeres. Las victorias de los movimientos feministas desde la década de los setenta, en parte legalizadas en la Constitución de Brasil de 1988, cambiaron el concepto oficial de la familia y la reproducción. Desde entonces el gobierno y la sociedad civil han desempeñado un papel fundamental para construir, tanto legalmente como en la práctica, una mayor igualdad y equidad entre hombres y mujeres. Sin embargo, tal y como lo muestran las discusiones que se dan en torno a las prácticas de esterilización, sigue existiendo una enorme brecha entre las victorias legales y las condiciones de vida reales.

${ }^{8}$ Aquí es importante mencionar que aunque algunos estudios sobre fecundidad sostienen que la fecundidad descendió desde principios del siglo XX. la mayoría continúa asegurando que el comienzo fue en la década de 1960 . Véase por ejemplo, Leone y Caetano (2002: 3). 
Perspectivas de los demógrafos brasileños sobre la fecundidad futura

Los descensos recientes de la fecundidad en Brasil, así como la práctica continua de la esterilización como mecanismo principal de control de la fecundidad, y su concentración en edades tempranas, llevaron a la comunidad demográfica al consenso de que la fecundidad en Brasil estará pronto por debajo del nivel de reemplazo. Hay poco desacuerdo entre los demógrafos brasileños respecto a que esto sucederá en la próxima década, aunque las proyecciones oficiales sostienen que las tasas globales de fecundidad estarán alrededor de los niveles de reemplazo hacia 2050. Hay diferentes puntos de vista respecto del ritmo y los niveles con que se podrían alcanzar dichas tasas; además no hay acuerdo sobre la irreversibilidad de esta tendencia o sobre el tiempo que se mantendrán por debajo del reemplazo.

El hecho es que el reciente y abrupto descenso de la tasa global de fecundidad -de 4.3 en 1980 a 2.2 en 2000 - ha venido acompañado por una creciente esterilización de las jóvenes brasileñas casadas, que aumentó de 4 a $11 \%$ entre las mujeres de 20 a 24 años, de 1986 a 1996 (Bemfam, 1997). También un considerable descenso en la mortalidad infantil de 116.9 a 44.1 entre 1970 y 2000 contribuyó a la baja de la fecundidad y al aumento de la esperanza de vida. Sin embargo, el gran incremento en la mortalidad de hombres jóvenes-de 15 a 34

\section{CUADRO 1}

Indicadores demográficos de Brasil establecidos y proyectados por su gobierno, 1970-2050

\begin{tabular}{|c|c|c|c|c|c|c|}
\hline \multirow[b]{2}{*}{ Indicadores } & \multicolumn{4}{|c|}{ Censos } & \multicolumn{2}{|c|}{ Proyecciones } \\
\hline & 1970 & 1980 & 1991 & 2000 & 2025 & 2050 \\
\hline Tasa global de fecundidad & 5.76 & 4.35 & 2.61 & 2.20 & 2.06 & 2.06 \\
\hline Mortalidad infantil & 116.9 & 69.1 & 44.1 & 33.8 & 24.2 & 15.1 \\
\hline $\begin{array}{l}\text { Esperanza de vida al } \\
\text { nacimiento }\end{array}$ & 52.7 & 61.8 & 65.9 & 68.6 & 71.0 & 73.6 \\
\hline $\begin{array}{l}\text { Porcentaje de menores } \\
\text { de } 20 \text { años } \\
\text { Porcentaje de mayores }\end{array}$ & 53.1 & 49.7 & 45.0 & 39.1 & 29.9 & 25.7 \\
\hline de 65 años & 3.1 & 4.0 & $\begin{array}{c}4.8 \\
46895\end{array}$ & 5.0 & $\begin{array}{l}9.6 \\
9.659\end{array}$ & $\begin{array}{c}16.2 \\
928169\end{array}$ \\
\hline
\end{tabular}

Fuente: Instituto Brasileño de Geografia y Estadística (IBGE), varios anuarios estadísticos, censos y proyecciones de población. 
años de edad-durante la década de 1980 debido a causas externas, limitó las ganancias en la esperanza de vida masculina, aumentando así la brecha de género. De 1991 a 2000 la esperanza de vida de los varones se incrementó de 62.6 a 64.8 años, mientras la de las mujeres pasó de 69.8 a 72.6 (IBGE, 2002).

Las tasas globales de fecundidad por debajo del reemplazo son ya parte de los regímenes demográficos de casi todas las zonas metropolitanas de Brasil, puesto que se registraron desde 1.8 en Belo Horizonte hasta 2.2 en Curitiba en 1999, cuando el promedio para Brasil era de 2.4 hijos por mujer. Siete de cada diez mujeres casadas que utilizan algún tipo de anticonceptivo en las áreas metropolitanas han elegido la esterilización de ellas o sus compañeros (Rodríguez Wong, 2001). Esta alta tasa de esterilización puede explicar la falta de correlación entre la fecundidad y la mortalidad infantil en las áreas metropolitanas. Por ejemplo, la zona metropolitana de Salvador (Bahia) tenía una de las más altas tasas de mortalidad infantil (62 por mil) y una de las tasas globales de fecundidad más bajas ( 1.8 hijos).

Las estimaciones y proyecciones recientes de las tasas globales de fecundidad por cohorte y periodo sugieren que la fecundidad en Brasil seguirá en descenso y que pronto alcanzará niveles de subreemplazo. La preocupación entre los demógrafos brasileños es que una exclusiva dependencia de la tasa global de fecundidad convencional basada en la edad (TGF) y las medidas de periodo podría generar confusión en el sentido de que las tendencias actuales fueran reales o bien, fluctuaciones de corto plazo, llevaron a la generación de tasas globales de fecundidad por cohorte. Los hallazgos más recientes confirman un descenso sistemático en la fecundidad por cohorte durante el siglo XX con tasas globales de fecundidad de $6.9,5.8$ y 4.2 para cohortes de mujeres según el año de inicio de su periodo reproductivo: 1903, 1933 y 1963 respectivamente (Horta et al., 2000). Otro estudio que proyecta las tasas globales de fecundidad por cohorte sugiere que aquellas situadas por debajo del reemplazo (1.9) serán alcanzadas por las mujeres que comenzaron su vida reproductiva en 1988. La proyección de la fecundidad en el mismo estudio muestra una tasa global de fecundidad de 2.0, 1.8, 1.6 y 1.5 para los años 2000, 2005, 2010 y 2015 , respectivamente (Carvalho et al., 2000).

De acuerdo con algunos demógrafos, la esperanza de que una baja fecundidad perdure en Brasil y lleve a un debilitamiento demográfico en el largo plazo, puede no estar garantizada. Ellos creen que se puede haber llegado al punto más bajo de un movimiento cíclico de 
largo plazo y que los cambios demográficos están entrando a un futuro completamente desconocido. Para algunos analistas el nivel promedio de la fecundidad sólo muestra una parte de la historia de la baja fecundidad. Siguiendo esta hipótesis y muy apegado a la interpretación del "lado de la demanda" de la fecundidad futura, un estudio basado en la encuesta DHS de 1996 señala la importancia del tamaño de familia ideal o deseada en diferentes regiones. Por ejemplo, el número ideal de hijos varía sólo de 2.4 en Río de Janeiro a 2.8 en São Paulo y en el Noreste, y la proporción de mujeres que declararon un ideal de dos hijos varía de $47 \%$ en Río a $37 \%$ en el Noreste. Además, un grupo importante de mujeres declaró que el número ideal de hijos era mayor que dos. En São Paulo 29\% declaró un ideal de tres hijos, y en el Noreste $28 \%$ deseaba cuatro hijos. El estudio también encontró que una alta proporción de mujeres del Noreste sin hijos, $24 \%$, no deseaba tenerlos, mientras que entre las que contaban con un solo hijo nacido vivo, cerca de $68 \%$ quería tener otro (Camarano et al., 1999).

Mientras estudiaba el tema de las perspectivas de la futura fecundidad en Brasil para realizar este artículo, apliqué una encuesta a un grupo de expertos demógrafos brasileños (EDB) ${ }^{9}$ en enero de 2002. Se formularon cinco preguntas, las cuales transcribo en los pies de página a lo largo del texto. Las primeras dos tienen que ver con la opinión de los EDB sobre las tendencias del comportamiento de la fecundidad en Brasil, incluyendo la posibilidad de que sus niveles lleguen a ubicarse por debajo de los del reemplazo. ${ }^{10}$

${ }^{9}$ Se identificó como expertos demógrafos brasileños (EDB) a aquellos que tenian un historial en el estudio de la fecundidad o en proyecciones de población. Consulté a diez personas que consideré EDB, de las cuales ocho respondieron, sugiriendo tendencias generales de la fecundidad en Brasil, $y$ seis de ellos proporcionaron estimaciones específicas de dichas tasas. Aquellos que respondieron fueron: Ana Amélia Camarano (AAC) del Instituto de Pesquisas de Economia Aplicada (IPEA), Eduardo G. Rios-Neto (ERV) del Centro de Desenvolvimento e Planejamento Regional (Cedeplar), Elza S. Berquó (EB) del Nucleo de Estudos de Populaçāo (NEPO), Fernando R. P. de Albuquerque (FRA) del Instituto Brasileiro de Geografia e Estatistica (1BGE), José Alberto M. de Carvalho (JAC) del Cedeplar, Kaizô Beltrāo (KB) de la Escola Nacional de Estatistica (ENCE), Laura Rodríguez Wong (LRW) del Cedeplar, y Sônia Corrêa (SC) del Instituto Brasileiro de Análises Sociais e Econômicas (IBASE).

${ }^{10}$ La pregunta 1 fue: "¿Seguirán las tasas globales de fecundidad en Brasil las tendencias de los llamados países desarrollados, y alcanzarán niveles por debajo del reemplazo, o se estabilizarán alrededor de los niveles de reemplazo?", y la pregunta 2 fue:

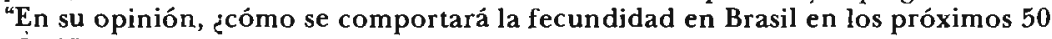
años?" 
La mayoría de los EDB estuvo de acuerdo en que Brasil podía seguir los patrones experimentados por países más desarrollados alcanzando niveles de fecundidad por debajo del reemplazo. Rios Neto sugirió que los países mediterráneos (Italia, Portugal, España y Grecia) son los modelos más cercanos a Brasil debido a sus raíces culturales comunes y a su tipo de "estado de bienestar", aunque deben ser consideradas las diferencias en los patrones de nupcialidad y de fecundidad. De manera similar, Sônia Corrêa afirma que la experiencia de los países mediterráneos es parecida a la de Brasil. Esta autora argumenta que estos países comparten el ethos católico y un patrón general de desigualdad de género, si se le compara con el de Europa del Norte. Además, en los países mediterráneos el apoyo del Estado para la salud reproductiva de la mujer y los programas de equidad de género son débiles, en lo cual se incluye la cuestión de la ilegalidad del aborto (con excepción de Italia, donde se legalizó desde la década de 1970). ${ }^{11}$

Algunos EDB defendieron más que otros la tendencia dirigida hacia los niveles de la fecundidad por debajo del reemplazo:

Con seguridad, la fecundidad en Brasil llegará a ubicarse por debajo del reemplazo (KB).

No hay duda de que las tasas globales de fecundidad de Brasil alcanzarán niveles por debajo del reemplazo en la primera década del siglo XXI (JAC).

Si bien las tasas globales de fecundidad de Brasil estarán por debajo del reemplazo, también estarán fluctuando (FRA).

Otros, aunque estaban de acuerdo con la tendencia dirigida hacia los niveles de la fecundidad por debajo del reemplazo, señalaron las particularidades y dificultades que presenta su estimación en Brasil:

Las tasas globales de fecundidad de Brasil seguirán la experiencia de los países desarrollados excepto por su dinámica peculiar de una fecundidad alta concentrada en edades tempranas. Los cambios dirigidos hacia un patrón de fecundidad tardía como el de los países desarrolla-

${ }^{11}$ En efecto, hacia finales del 2001 seis mujeres fueron condenadas por el crimen de aborto en Portugal. 
dos son posibles en un mediano plazo, aunque ello no está garantizado (ERN).

En cierto punto en el tiempo, no hay duda de que tendremos tasas globales de fecundidad por debajo del reemplazo. Aun siendo conservador, yo diría que esto ya está sucediendo para 30 o $40 \%$ de la población brasileña. Para el país entero, es posible que dichos niveles se alcancen en los próximos cinco años. Además, dado que Brasil todavía tiene subgrupos de población con rangos de mortalidad relativamente altos, una tasa global de fecundidad por encima de 2.1 puede estar implicando ya para ellos tasas de crecimiento intrínsecas por debajo del cero (LRW).

Es difícil pensar en una fecundidad por debajo del reemplazo, ya que la heterogeneidad regional oculta las tendencias de la fecundidad promedio del país. Mientras las tasas globales de fecundidad en regiones como São Paulo y Río de Janeiro parecen acercarse a los niveles por debajo del reemplazo, no creo que el Noreste, una gran región con las tasas globales de fecundidad más altas, alcance dichos niveles (AAC).

Si tomamos un ejemplo regional como Rio de Janeiro, no parece absurdo pensar en una fuerte tendencia hacia un "sub-reemplazo". Además, deberíamos considerar el peso relativo del fenómeno "oleadas de juventud" en la estructura de la población brasileña y su contribución potencial para las proyecciones futuras de población (SC).

\section{Pronosticando tasas globales de fecundidad: 2000-2050}

En la pregunta acerca del comportamiento de la fecundidad en Brasil en los próximos 50 años, seis de los ocho expertos demógrafos brasileños respondieron que las tasas globales de fecundidad continuarán descendiendo hacia niveles por debajo del reemplazo, mientras que uno cree que la fecundidad oscilará alrededor de los niveles de reemplazo. Dos del grupo de seis consideraron la posibilidad de que se presente un ligero aumento al término de los 50 años, aunque no esperan que se vuelva a alcanzar el nivel de reemplazo.

Cuando se les pidió que especificaran las tasas globales de fecundidad promedio para tres periodos durante los próximos 50 años, sólo respondieron seis de los ocho EDB. ${ }^{12} \mathrm{He}$ aquí sus respuestas:

${ }^{12}$ La pregunta 3 fue: “¿Cuál es su estimación de la tasa global de fecundidad para los siguientes periodos: 2000-2005, 2005-2025, 2025-2050?" 


\begin{tabular}{lllllll}
\hline Años & $J A C$ & $E R N$ & $L R W$ & $F R A$ & $K B$ & $A A C$ \\
\hline $2000-2005$ & 2.0 & 1.9 & 2.1 & 2.3 & 2.4 & 2.1 \\
$2005-2025$ & 1.8 & 1.3 & 1.8 & 2.1 & 1.9 & 1.7 \\
$2025-2050$ & 1.8 & 1.7 & 1.5 & 1.9 & 1.6 & - \\
\hline
\end{tabular}

Estos demógrafos, atendiendo a lo que se les solicitó, justificaron sus pronósticos ${ }^{13}$ ofreciendo una combinación de razonamiento sustantivo y metodológico. El supuesto del descenso como una tendencia pasada es común como explicación de las tasas estimadas para el periodo 2000-2005 (KB, AAC, ERN, JAC, FRA). Otras justificaciones incluyeron la convergencia del comportamiento entre grupos sociales y regiones (JAC, FRA), el incremento en el nivel educativo de las mujeres -que está altamente correlacionado con las tasas de fecundidad(ERN), y una situación socioeconómica precaria y continua para amplios segmentos de la población (LRW).

Para el periodo de 2005-2025 la mayoría de los EDB estimó tasas por debajo del reemplazo. Dichas estimaciones son resultado de "un modelo de regresión logística de proyección" ( $\mathrm{AAC}, \mathrm{KB}$ ), o de la convergencia de comportamientos, ya que "las tasas de fecundidad entre los grupos de ingreso medio y alto eran ya de 2.1 en 1970" (JAC). Otras justificaciones atienden al hecho de que sería difícil revertir la tendencia que va por debajo del reemplazo "sin cambios estructurales y en ausencia de una fuerte intervención de política pública llevada a cabo mediante una creciente mezcla anticonceptiva que buscara principalmente el espaciamiento entre los nacimientos en lugar de poner fin a la fecundidad" (ERN, LRW). Para el periodo de 2025-2050 las tasas de fecundidad podrían aumentar, descender o permanecer estables respecto del periodo previo. Algunos creen que una potencial recuperación de las tasas (no necesariamente alcánzando los niveles de reemplazo) podría ser resultado de ciertas políticas públicas de largo plazo, pero especialmente debida a factores externos asociados al sistema de seguridad social y al mercado de trabajo (ERN); o bien sería consecuencia de la globalización de los sistemas masivos de comunicación y de la economía (LRW, KB).

${ }^{19}$ La pregunta 4 de la encuesta fue: “¿Por qué piensa que éste será el promedio de los comportamientos reproductivos esperados en cada uno de los periodos?" 
Proyecciones de población para Brasil: los escenarios de la División de Población de las Naciones Unidas

Al considerar el razonable y sólido supuesto de la futura fecundidad utilizado por la División de Población de las Naciones Unidas (UNPD por sus siglas en inglés) al elaborar sus proyecciones para Brasil, mi primera conclusión es que su variante o hipótesis media acerca de la velocidad del descenso de la fecundidad es demasiado cauta. La estimación de la UNPD para los próximos 50 años, en la variante o hipótesis media, es mayor que la esperada por los expertos demógrafos brasileños y que la arrojada por los hallazgos recientes. Si la fecundidad es un factor determinante en el crecimiento de la población de Brasil, entonces la variante o hipótesis baja de la UNPD se adecua mejor a la situación del país.

Dicha variante o hipótesis media supone tasas globales de fecundidad cercanas al nivel de reemplazo, que varian de 2.15 a 2.10 en el curso del periodo 2000-2050. En esta "variante" se espera que la mortalidad infantil disminuya sistemáticamente, de 38 a 8 por mil, y que la esperanza de vida al nacimiento se incremente de 68 a 77 años para ambos sexos. La migración neta se considera nula y las tasas de crecimiento disminuirán de 1.22 a $0.32 \%$ anual entre 2000 2050.

Conforme a lo anterior, casi todos los EDB estarían de acuerdo con la UNPD en que la tasa general de fecundidad en Brasil estará cercana al nivel de reemplazo en el periodo de 2000 a 2005. Sin embargo la mayoría también espera que se alcancen niveles por debajo del reemplazo para 2025, al contrario de la expectativa de la UNPD, que supone su estabilización. Estas opiniones se refuerzan con los hallazgos de tasas globales de fecundidad por debajo del reemplazo en casi todas las áreas metropolitanas -que juntas representan entre $30 \mathrm{y}$ $40 \%$ de la población del país- en el año 2000 . Por consiguiente, las principales diferencias entre los escenarios presentados por la UNPD (variante o hipótesis media) y los demógrafos brasileños se derivan de un ritmo distinto del cambio en la fecundidad y del crecimiento de la población en sus respectivas proyecciones. Como ya expuse, la variante o hipótesis baja de la UNPD para Brasil, con su tasa global de fecundidad por debajo del reemplazo de 1.83 en el periodo inicial de 2000 2005 , seguido de una TGF de 1.6 para 2050 corresponde mejor a los hallazgos recientes y a las opiniones de la mayoría de los expertos demógrafos brasileños. 
Las bajas tasas de crecimiento poblacional de Brasil son resultado principalmente del descenso de la fecundidad. Aun cuando el aumento en la esperanza de vida al nacimiento -de 49.3 a 67.0 años entre 1950-2000- ha contribuido al crecimiento de la población, ha sido mayor el efecto de la baja en la fecundidad que el de la esperanza de vida creciente. A pesar de la caída de las tasas de crecimiento, se espera que continúe el aumento poblacional debido a las altas tasas del pasado. Por consiguiente, la población de Brasil continuará incrementándose a pesar del descenso de la fecundidad. En la más reciente revisión de las proyecciones de la UNPD para 2050 se estimó una población total, en la variante o hipótesis media, de 247 millones, o un incremento de $45 \%$ con relación al año 2000.

Debido a que las estimaciones medias de la fecundidad realizadas por la UNPD eran tan diferentes a los hallazgos recientes y a las opiniones de los demógrafos brasileños, decidí examinar otras proyecciones recomendadas para Brasil. Comparé la variante o hipótesis media de la UNPD con la proyección de la Oficina del Censo de Estados Unidos (USCB, por sus siglas en inglés) para Brasil, pues ésta supone que la fecundidad alcanzará niveles por debajo del reemplazo en 2005, lo cual es más cercano a los hallazgos recientes y a las opiniones de la mayoría de los expertos demógrafos brasileños. Además, las dos organizaciones utilizaron fuentes de información y procedimientos similares para estimar las variables demográficas. ${ }^{14}$

${ }^{14}$ Las Naciones Unidas y la Oficina del Censo de Estados Unidos se basan en las mismas fuentes de información y generalmente utilizan técnicas similares para estimar los parámetros demográficos. Las Naciones Unidas realizan nuevas estimaciones cada año y la Oficina del Censo revisa sus proyecciones una vez al año y actualiza su base de datos internacional al menos dos veces al año. Existen diferencias en los softwares que se utilizan para hacer las proyecciones. Sin embargo, la USCB aclara por qué sus proyecciones difieren de las de Naciones Unidas: "no es probable que las diferencias significativas en las proyecciones de población para 20 o 30 años en el futuro se atribuyan al sofiware. Cuando se trata de algo más que diferencias pequeñas entre las proyecciones es más probable que se deban a: 1) diferencias en la disponibilidad de la información del país para las dos organizaciones; 2) diferencias en la evaluación de la calidad de los datos y en las estimaciones basadas en la información del país y hechas por los equipos de analistas de Naciones Unidas y de la USCB; 3) diferencias en los protocolos específcos de las instituciones en términos de la forma en que se hacen las proyecciones de fecundidad, mortalidad y migración" (U.S. Census Bureau, 1999: B-16-17). 


\section{¿En qué difieren las proyecciones para Brasil de la División de Población de las Naciones Unidas y las elaboradas por la Oficina del Censo de Estados Unidos?}

El contraste entre las proyecciones de la UNPD y las del USCB para la población total de Brasil es sorprendente. Para 2050 la diferencia en la población estimada de Brasil es $20 \%$ o 40.5 millones de personas más en las proyecciones de la UNPD respecto de las del USCB. A pesar de que ambas parten de valores iniciales similares para la fecundidad total $(\mathrm{TGF}=2.1)$, plantean distintos supuestos sobre el ritmo y el nivel del descenso de la fecundidad a lo largo de los 50 años que van de 2000 a 2050. Como puede observarse en el cuadro 2, el USCB supuso que la TGF de Brasil estará por debajo del nivel de reemplazo desde 2005 y que perdurará una tasa de 1.7 en el periodo 2025-2050. La UNPD supone que la fecundidad se mantendrá en los niveles de reemplazo (TGF $=2.1$ ) a lo largo de 2000-2050. A pesar de que siempre suelen intervenir elementos subjetivos en la elección del modelo, para los primeros años los supuestos son relativamente "objetivos" y las proyecciones producen resultados similares. En contraste, para los años más distantes todas las predicciones de población implican juicios, lo que las hace particularmente subjetivas.

Además, la UNPD y el USCB proyectan estructuras de edad distintas en diferentes intervalos. De acuerdo con la proyección de la UNPD, $47.2 \%$ de la población brasileña estará para 2025 en las edades “dependientes" (menos de 20 y más de 65 años de edad) en comparación con $34.7 \%$ previsto por la proyección del USCB. Esta diferencia de $12.5 \%$ o de más de 20 millones de personas se debe, sobre todo, a los jóvenes dependientes ( 10 del $12.5 \%$ de diferencia), y aparentemente resulta de la mayor tasa global de fecundidad proyectada por la UNPD.

Aun cuando la tasa global de fecundidad desempeña un papel crucial en las proyecciones, pueden también observarse diferencias en los supuestos sobre mortalidad entre las dos organizaciones. Tales supuestos se establecieron en términos de incrementos en la esperanza de vida al nacimiento por década, y las diferencias parecen reflejar diferentes perspectivas sobre futuros desarrollos que afectarán la mortalidad y su calendario. Al parecer, el USCB considera, al contrario de las creencias pasadas, que hoy existe un grado considerable de incertidumbre sobre el futuro de la mortalidad. En países en desarrollo como Brasil esto se deriva del futuro incierto de la epidemia de sida y 
GRÁFICA 1

Proyecciones de población para Brasil realizadas por la USCB y la UNPD: 2000-2050

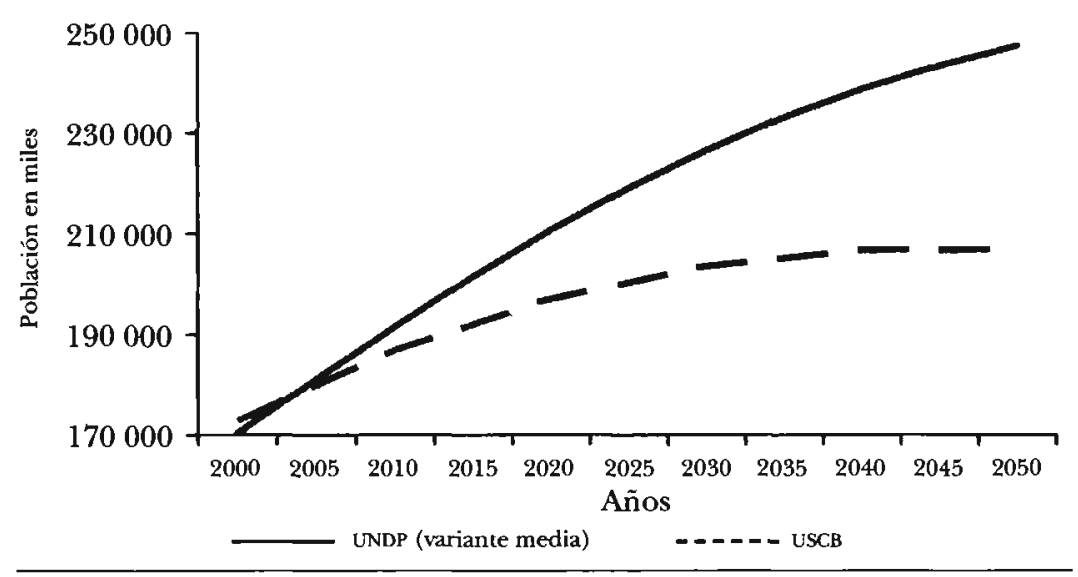

de otras enfermedades infecciosas, así como del desarrollo de los servicios de salud. En países industrializados la incertidumbre está más asociada a la disputa de si ya estamos cerca o no del límite biológico de la esperanza de vida. En consecuencia, el USCB estima una menor esperanza de vida para 2000 y 2025 (62.9 y 70.5 respectivamente) comparada con la estimada por la UNPD ( 68.3 y 73.0 respectivamente). Sin embargo, después de 2025 el USCB espera ganancias en la esperanza de vida, con lo que rebasaría las estimaciones de la UNPD. De este modo, los supuestos de la esperanza de vida para 2050 son 79.9 para el USCB y 76.9 para la UNPD.

De este modo, el valor inicial de la esperanza de vida al nacimiento para ambos sexos es de $\mathbf{5 . 4}$ años más en las proyecciones de la UNPD; para 2025 la UNPD sigue prediciendo por delante con 2.5 años de ventaja respecto de las proyecciones del USCB. El supuesto del USCB en relación con los retrasos en la mortalidad en Brasil para el periodo 2000-2025 contribuye a reducir la población total comparada con las estimaciones de la UNPD, lo cual merece una discusión especial que no es posible presentar aqui. Cabe aclarar que mientras muchos estudios sobre mortalidad en Brasil apoyan las proyecciones de la UNPD, los hallazgos recientes respaldan las proyecciones del USCB. Como se mostró en el cuadro 1, el gobierno de Brasil, por medio de las estimaciones del Instituto Brasileño de Geografia y Estadística (BBGE), 
CUADRO 2

Indicadores demográficos de Brasil para 2000, 2025 y 2050, de acuerdo con las proyecciones de la División de Población de Naciones Unidas y de la Oficina del Censo de Estados Unidos

\begin{tabular}{lrrrrrrrr}
\hline & \multicolumn{3}{c}{$\begin{array}{c}\text { División de Población } \\
\text { de Naciones Unidas }\end{array}$} & & \multicolumn{3}{c}{$\begin{array}{c}\text { Oficina } \\
\text { del Censo de EU }\end{array}$} \\
\cline { 2 - 3 } Indicadores & 2000 & 2025 & 2050 & & 2000 & 2025 & 2050 \\
\hline Tasa global de fecundidad & 2.15 & 2.10 & 2.10 & & 2.13 & 1.71 & 1.70 \\
Mortalidad infantil & 38.0 & 22.0 & 8.0 & 38.0 & 17.4 & 7.5 \\
Esperanza de vida al nacimiento & 68.3 & 73.0 & 76.9 & 62.9 & 70.5 & 79.9 \\
Porcentaje de menores de 20 años & 38.9 & 37.0 & 26.5 & & 39.2 & 27.0 & 21.7 \\
Porcentaje de mayores de 65 años & $\mathbf{5 . 1}$ & $\mathbf{1 0 . 2}$ & 17.9 & & 5.3 & 7.7 & 21.1 \\
\hline
\end{tabular}

reconoce el impacto de la creciente mortalidad de hombres jóvenes en la esperanza de vida; dichas estimaciones proyectan una cifra que es casi la misma prevista por el USCB para 2050. De manera similar Beltrão y Camarano (2001) proyectan esperanzas de vida al nacimiento menores que las pronosticadas por el USCB y el IBGE. Por ejemplo, estiman una esperanza de vida de 62 años para los hombres y de 68 años para las mujeres en 2025, aun cuando dejan abierta la posibilidad de que se logren mejoras en la esperanza de vida para las siguientes décadas.

Los efectos de las distintas proyecciones son particularmente evidentes en la estructura por edad. La proporción de adultos mayores dependientes se triplica de 7.7 a 21.1 en las proyecciones del USCB entre 2025 y 2050 , mientras que apenas se incrementa 3.1 puntos porcentuales en las proyecciones de la UNPD, lo que claramente refleja los efectos del régimen sostenido de fecundidad por debajo de los niveles de reemplazo planteado por el USCB. Carvalho argumenta que aunque la mortalidad no desempeñará un papel importante en el futuro de la fecundidad en Brasil, las ganancias en la mortalidad en las próximas décadas se darán sobre todo entre las personas de edades avanzadas, lo cual podría significar un crecimiento todavía más rápido de la proporción de adultos mayores en la población de Brasil (JAC).

Las más recientes proyecciones para Brasil parecen reforzar las evaluaciones previas en el sentido de que las proyecciones de la UNPD son buenas en estratos muy agregados, aunque menos precisas para todo el país. Entiendo que las proyecciones para Brasil son un pequeño subconjunto de las correspondientes a la población global, y que 
por ello es difícil para la División de Población de las Naciones Unidas tomar en cuenta las opiniones de los expertos y las especificidades de las tasas demográficas de cada país. Sin embargo, espero que la opinión de la comunidad de demógrafos de Brasil pueda servir a la UNDP para construir supuestos más cercanos a la realidad en sus proyecciones. También me parece pertinente reconocer la relevancia del debate sobre las proyecciones probabilísticas y la importancia de conceder mayor atención a la incertidumbre en las proyecciones. Evidentemente las evaluaciones erróneas no mejoran la precisión de los pronósticos, pero al reconocer de manera adecuada la incertidumbre se incrementa la posibilidad de producir pronósticos por intervalos que reflejen de forma más apropiada unos desarrollos futuros de la fecundidad más razonables (Alho, 1997; Lutz et al., 1998).

\section{Las fuerzas que darán forma a la fecundidad en Brasil}

Casi toda la literatura demográfica sobre Brasil y las opiniones de los expertos demógrafos brasileños coinciden en que lo más probable es que la herencia demográfica y el actual comportamiento reproductivo determinen la fecundidad del país para las próximas décadas. ${ }^{15}$ Además, existe consenso en el sentido de que las futuras leyes y políticas relacionadas con la esterilización, la equidad de género y el apoyo público también darán forma a la fecundidad futura de Brasil.

\section{Legado demográfico y comportamiento reproductivo actual}

En el 2000, 39\% de la población de Bras̀il tenía menos de 20 años, lo que resulta en parte de la "oleada joven" que fue procreada por un gran número de madres nacidas durante el periodo de alta fecundidad. ${ }^{16}$ Estos jóvenes, que a principios de la década de 1990 cumplieron de 15 a 24 años, han reducido su fecundidad, contribuyendo así a los actuales niveles cercanos a los de reemplazo (TGF $=2.3$ ). La fecun-

${ }^{15}$ La pregunta 5 fue: “En su opinión, ¿cuáles son los factores o los principales indicadores que podrian ser particularmente relevantes y útiles para formular supuestos plausibles en relación con el futuro de la fecundidad en Brasil?"

${ }^{16}$ El "boom de jóvenes" es el resultado de $66 \%$ de incremento en las cohortes nacidas entre 1965 y 1980 en relación con las cohortes previas (Bercovich y Madeira, 1999/2000). 
didad experimentó un proceso de rejuvenecimiento de 1970 a 1988 , cuando las personas de 15 a 24 años representaban $30 \%$ de los nacimientos al inicio del periodo y $45 \%$ al final. De 1988 a 2000 la proporción permaneció constante. Al mismo tiempo, como ya expusimos, la esterilización se incrementó entre las jóvenes casadas (20 a 24 años) de 4 a $11 \%$ entre 1986 y 1996. Las intenciones reproductivas de las mujeres brasileñas así como sus prácticas reproductivas son sorprendentes. En 1996 alrededor de $80 \%$ de las mujeres brasileñas casadas y en edad reproductiva no podían o no querían tener más hi$\operatorname{jos}^{17}$ y $44 \%$ de las mujeres que no utilizaban método anticonceptivo alguno consideraban que la esterilización podria ser un método aceptable para controlar su fecundidad. De manera conjunta estos indicadores demográficos sugieren una trampa en la fecundidad, en la cual los niveles de reemplazo son el destino seguro.

Los demógrafos brasileños creen que entre las fuerzas capaces de cambiar el ritmo de los niveles y el calendario de la fecundidad en Brasil se encuentran los cambios en la prevalencia de la "mezcla" de métodos anticonceptivos, los nacimientos fuera del matrimonio, y cualquier otra variable que pudiera reducir el tiempo de exposición al riesgo de concebir, tales como la edad mediana al contraer matrimonio y al iniciar la cohabitación (LRW, JAC, ERN, KB). En relación con esto último es importante advertir que aunque la fecundidad es mayor entre las mujeres que cohabitan que entre aquellas que están legalmente casadas, durante el periodo 1976-1984 se registró un incremento en el índice limitativo de las parejas cohabitantes (medida de control de la fecundidad después de 10 años de cohabitación), lo que dio como resultado la convergencia de las tasas globales de fecundidad de las mujeres casadas y de aquellas que viven en unión libre (Lazo, 1994).

Las crecientes tasas de inestabilidad marital ilustran las expectativas cambiantes acerca de la permanencia del matrimonio en Brasil. La cohabitación se incrementó de 18 a $28 \%$ entre los censos de 1991 y de 2000 , y la proporción de mujeres en edades reproductivas con más de una unión cambió de alrededor de 10 a $13 \%$ en el periodo de 1986 a 1996. Las tasas de separación y divorcio se han duplicado en las últimas dos décadas, y las recientes reformas a las leyes de divorcio y custodia han reforzado tales comportamientos y actitudes cambian-

${ }^{17}$ Esta proporción está constituida más o menos por $43 \%$ de mujeres esterilizadas, $4 \%$ de mujeres infértiles, $12 \%$ de mujeres sin hijos que no quieren tenerlos y $21 \%$ de mujeres con hijos que ya no quieren tener más. 
tes. Las parejas casadas tienen ahora menos hijos, y un creciente número de mujeres está criando a sus hijos fuera de matrimonio. A principios de la década de 1990 , más de $15 \%$ de todos los partos en Brasil correspondían a madres no casadas.

El impacto potencial de una creciente fecundidad adolescente y la inestabilidad marital en las tasas globales de fecundidad sugiere que la baja en la fecundidad habría sido más importante en ausencia del incremento del embarazo adolescente y la inestabilidad de las uniones $^{18}$ (Leone y Caetano, 2002). Los hallazgos muestran que entre 1986 y 1996 la tasa global de fecundidad parece haberse incrementado $2.4 \%$ debido a la fecundidad adolescente, manteniéndose constante el patrón de uniones y los niveles de esterilización. Si las tasas de fecundidad de las mujeres con una sola unión se hubieran aplicado a todas, la tasa global de fecundidad habría sido $3.2 \%$ mayor en 1996, manteniendo constante la tasa de fecundidad adolescente y la esterilización a los niveles de 1996. Al mismo tiempo, la medida de la magnitud del efecto de la esterilización en la fecundidad muestra que en la mitad de la década de 1980 la tasa global de fecundidad era 17\% menor de lo que habría sido, y que a mediados de la década de 1990 el efecto de la esterilización en la baja de fecundidad se había incrementado hasta $26 \%$. Por consiguiente, Leone y Caetano concluyen que la difusión de la esterilización femenina excedió los efectos positivos de las otras dos variables, contribuyendo así a reducir la TGF del país.

Finalmente, una menor fecundidad permite que las mujeres participen de manera más amplia en las actividades fuera del hogar, y en la mayoría de los casos ellas han venido encontrando menos atractivo el trabajo diario "de guardia" que representa ser madre (Presser, 1995), de ahí que el efecto positivo del descenso de la fecundidad en la vida de las mujeres y en la posibilidad de regresar a la familia tradicional, aquella del tipo "proveedor-ama de casa" se convierta en una cuestión central para pensar el tema del futuro de la fecundidad. La investigación cuantitativa y cualitativa da cuenta de la permanencia de las brasileñas de distintos estratos sociales en el mercado de traba-

${ }^{18}$ La información de este estudio proviene de las encuestas DHS de 1986 y 1996 , cuyos resultados muestran: 1) un incremento en la tasa de fecundidad adolescente de 15 a 19 años, de 74.2 a 86.3 ; 2) una disminución de la edad media al primer nacimiento, de 20.9 a 19.8 , y 3) un aumento de las mujeres casadas al menos una vez (de 15 a 49 años) con más de una unión, de 9.6 a $13.3 \%$ (Leone y Caetano, 2002). 
jo y de las mejoras de las mujeres en educación y en otras esferas públicas, lo cual sugiere la improbabilidad de un regreso al modelo tradicional de familia del "proveedor-ama de casa" como estímulo para cambiar la dirección del descenso de la fecundidad. A pesar de que están conscientes de los costos físicos y emocionales del doble cambio, las brasileñas no quieren dejar su empleo aun cuando a veces la remuneración es modesta, dado que ello les ha permitido disponer de mayor independencia y poder de negociación en sus hogares (Sarti et al., 1990). Aun cuando ellas deseen tener hijos, pueden terminar decidiendo tener menos o no tener ninguno. Esto es especialmente cierto para las mujeres jóvenes, ya que con mayor educación que las generaciones previas, cuentan con más información sobre sexualidad y anticoncepción, mayores oportunidades en el mercado de trabajo y más posibilidades de obtener independencia económica.

\section{La ley de esterilización ${ }^{19}$ y las políticas públicas}

A pesar de los esfuerzos del movimiento de mujeres y de las frustradas iniciativas de los servicios públicos de planificación familiar para ofrecer una amplia gama de opciones de métodos anticonceptivos, a mediados de la década de los noventa las mujeres brasileñas se siguen enfrentando a una elección dramática: "hacerse un aborto clandestino, hacerse esterilizar o continuar un embarazo no deseado" (Berquó, 1999: 125). Las altas tasas de mortalidad materna (114 defunciones por 100000 nacidos vivos en 1991) y las crecientes tasas de esterilización, ambas relacionadas con un uso abusivo de las cesáreas, son indi-

${ }^{19}$ La ley de esterilización fue aprobada en agosto de 1997 y establece: 1) La esterilización como un derecho para hombres y mujeres mayores de 25 años. 2) Las personas que busquen la esterilización a través del sistema público deben esperar 60 días después de la solicitud, periodo en el cual serán aconsejadas sobre las opciones de métodos anticonceptivos y los efectos secundarios potenciales de la esterilización. 3) Las esterilizaciones post-parto (dentro de las $\mathbf{4 8}$ horas después del parto) serán autorizadas sólo en el caso en que exista una indicación médica, como por ejemplo, una historia de cesáreas múltiples. El Ministerio de Salud instrumentó la legislaciôn regulatoria para que los hospitales públicos proporcionaran los servicios de esterilización, incorporando los procedimientos quirúrgicos en su lista de procedimientos médicos reembolsables, otorgando a los estados y municipios la responsabilidad de autorizar a los serviciós del sistema público para realizar oclusiones tubarias, y haciendo cumplir la ley. Por tanto, los hospitales autorizados son las únicas unidades que cuentan con permiso para recibir pago por el procedimiento de esterilización (Ministério da Saúde, 1997). 
cadores de las dificultades que ha implicado la instrumentación de programas de salud para la mujer. Sin embargo es importante advertir que en la segunda mitad de la década de 1990 hubo cierto progreso en el cuidado prenatal, en la atención de tipo obstétrico, y en el acceso a aborto en los casos de violación y de que esté en riesgo la vida de la madre (Corrêa et al., 1998).

La definición constitucional de planificación familiar como un derecho y una responsabilidad del gobierno de Brasil fue establecida en 1988. No obstante, la ley ordinaria que regula esta definición constitucional no fue aprobada sino hasta nueve años más tarde. En 1997 se adoptó una ley que establece normas claras con relación a los procedimientos para la esterilización, aunque es probable que no sea instrumentada antes de cinco años, según muestra la experiencia. Corrêa (2001) considera que "las distorsiones en la prevalencia anticonceptiva (y el alto porcentaje de cesáreas asociadas con esterilización) cristalizadas debido a diez años de retraso en la política pública no son fáciles de deshacer". En efecto, un estudio reciente confirma que la instrumentación de la ley de esterilización está lejos de lo deseable. Esto lleva a Berquó y Cavenaghi (2002) a concluir que "la ley ha cambiado poco la práctica común de la esterilización y hasta ahora no satisface los derechos reproductivos individuales".

La influencia del Estado brasileño en el comportamiento reproductivo ha sido ampliamente discutida (Goldani, 2001). Algunos de los expertos demógrafos brasileños coinciden en que el papel del Estado y de las políticas públicas será crucial para revertir la tendencia que va hacia niveles de la fecundidad de sub-reemplazo. Sin una seria inversión del Estado brasileño para modificar las condiciones estructurales imperantes para reducir la fecundidad, y para desarrollar un conjunto de políticas "amigables" para las mujeres, será muy dificil revertir las tendencias actuales (ERN, SC). La incertidumbre de los efectos de las políticas públicas incluye la incertidumbre acerca del momento de su instrumentación, de los recursos que se dedicarán a ese fin, y de la aceptación pública, como en el caso de la legalización del aborto.

Los efectos de la ley de esterilización (1997) en el sentido de mantener una "fecundidad potencial" entre las mujeres jóvenes sólo serán exitosos si se acompañan, de manera consistente, de una política efectiva de incremento en el acceso a métodos contraceptivos reversibles, lo cual está apenas comenzando a suceder. Además, es importante reconocer que los niveles de esterilización en Brasil no serían los mismos si el aborto no 
fuera ilegal y riesgoso. Por tanto, en un contexto marcado por una "cultura de la esterilización", una creciente oferta de métodos reversibles debiera asociarse con un amplio acceso al aborto a fin de garantizar un respaldo cuando los métodos reversibles fallen. El "tiempo oportuno de la política pública" se convierte entonces en un factor crucial, porque la experiencia brasileña ha sido de grandes retrasos respecto de la instrumentación adecuada de las leyes y los programas de salud requeridos. Considérese que la ley de esterilización de 1997 no está siendo efectiva por completo. Por consiguiente, aun en un ambiente social y de política pública más favorable, lo cual parece ser el caso de Brasil, los impactos o las influencias de buenas políticas de salud reproductiva no serán visibles antes de cinco años. De acuerdo con lo anterior, la posibilidad de legalizar el aborto y de obtener un apoyo público universal para que las mujeres hagan frente a la carga de la reproducción social es todavia remota. Tambièn resulta inquietante notar que ninguna de estas medidas tendrá efectos sin un esfuerzo sólido para producir una equidad de género (SC).

En consecuencia, no se está respondiendo a las necesidades ni a las garantías de los derechos reproductivos de millones de mujeres brasileñas. Además, Brasil tiene que hacerle frente al incremento de $15 \%$ en el tamaño de la población en edad reproductiva en las próximas dos décadas. Hay una creciente preocupación derivada de que la provisión de productos de salud reproductiva para la planificación familiar, la maternidad segura y la prevención y tratamiento de las enfermedades sexualmente transmisibles serán cada vez más escasos debido a los problemas económicos y las desigualdades, y a las reducciones de la ayuda internacional. O, como bien apunta Corrêa (2001), "el mayor problema que enfrentamos globalmente no es la escasez de recursos per se, sino el reto de una desviada distribución de recursos entre hombres y mujeres, entre Norte y Sur, y entre los sectores privado y público". Por tanto, los esfuerzos para movilizar los recursos públicos y privados y para construir asociaciones que se propongan atender esas preocupaciones son cruciales. El gobierno de Brasil debe mejorar su desempeño al dirigir sus prioridades de política social para evitar los retrasos en la optimización de las condiciones de salud y el descenso de la mortalidad.

\section{Las relaciones de género y los sistemas puiblicos de apoyo}

El efecto de la extraordinaria permanencia de la familia y la jerarquía de género en el descenso de la fecundidad es una cuestión intrigante y crucial que casi no ha sido considerada en las múltiples explicacio- 
nes sobre la baja de la fecundidad en Brasil (Goldani, 2001). Un estudio sobre el Noreste que utilizó información de la encuesta DHS de 1991 abordó el tema de los efectos de la igualdad de las relaciones esposo-esposa en la tasa global de fecundidad. En dicho estudio se encontró que los niveles de igualdad en relación con las elecciones reproductivas eran muy bajos -sólo $52 \%$ de las parejas tenían al menos algo de igualdad-y que para cada incremento de un punto en la escala de igualdad -donde el valor más alto era de tres puntos-, había una reducción de casi medio hijo (-0.570) entre las parejas con la misma duración de unión y número ideal de hijos (Goldani, 1999-2000). Hallazgos basados en la encuesta DHS de 1996 sugieren que aunque en promedio las metas de la fecundidad de los hombres no difieren mucho de las de sus esposas, ${ }^{20}$ el proceso de negociación en un matrimonio para resolver diferencias puede generar resultados que son sistemáticamente mayores o menores que los que están basados sólo en las percepciones de cualquiera de los esposos. Además, el autor de ese estudio concluyó que en la mayoría de los países de América Latina no hay evidencias de que las preferencias de los hombres tiendan a prevalecer por encima de las de sus esposas en lo que se refiere a definir el resultado final de su fecundidad. Reconoce que esto varía entre los países: en Bolivia sí ocurre; en Brasil esta tendencia sería insignificante $y$, en República Dominicana la tendencia sería la contraria (o sea, las preferencias de las mujeres dominicanas tienden a prevalecer por encima de las de sus esposos en lo que se refiere a definir el resultado final de su fecundidad) (Hakkert, 2001).

Desde una perspectiva masculina de la reproducción, un estudio de dos generaciones de hombres brasileños de clase media muestra que sus prácticas anticonceptivas pueden entenderse mejor como parte de la dinámica entre géneros. La importancia del condón y del método del ritmo entre las clases medias de la más grande metrópoli de Brasil (São Paulo) se considera un hallazgo sorprendente, aunque resulta consistente con las respuestas de los encuestados, particularmente de los miembros de las cohortes más jóvenes que creen que la píldora es dañina para la salud de las mujeres. Aunque las generacio-

${ }^{20}$ En la encuesta DHS de 1996 el número promedio ideal de hijos para aquellas parejas brasileñas donde al menos uno de los integrantes declaró un número definitivo, resultó en alrededor de 2.6 para la esposa y 2.9 para el esposo. Sin embargo, si los desacuerdos entre esposos se resolvieran sistemáticamente adoptando los números mayores para el ideal de hijos de la pareja, se alcanzaría un promedio de 3.4 hijos, pero si se escogiera el número más pequeño, el promedio de hijos sería 2.0 (Hakkert, 2001: 20). 
nes más jóvenes muestran señales de cambio, los autores enfatizan la necesidad de llevar a la práctica programas educacionales con sensibilidad de género dirigidos a los hombres. Estos programas deben ayudar a que se negocie mejor el uso de la anticoncepción (Oliveira et al., 2001).

Las mujeres brasileñas, que son jefas de una cuarta parte del total de hogares y que representan casi la mitad de la fuerza de trabajo remunerada, han logrado importantes ganancias en la esfera pública; sin embargo esto ha tenido poco efecto en las decisiones individuales y familiares que afectan sus vidas: la negociación de las relaciones de género sigue siendo difícil aun para las mujeres con trabajo remunerado. En 1996, de cada 100 mujeres casadas que trabajaban y tenían hijos menores de cinco años, 23 cuidaban a sus hijos, y los esposos sólo ayudaban en 4 de los casos. En $46 \%$ de los casos, otros parientes eran los principales responsables de ayudar a estas mujeres, ${ }^{21}$ mientras que el resto de ellas apoyaba en otros arreglos, particularmente en el servicio de guardería privado. El incremento de las familias con dos fuentes de ingresos -que representaban $52 \%$ de las familias en 1995-, y las familias de un padre o madre solo y empleado, ha generado mayor preocupación por la calidad de las medidas de protección al empleo, tales como la incapacidad por maternidad, las políticas de equidad en los salarios, y los servicios de cuidado de los niños. Dado que ser madre trabajadora se ha vuelto la norma, es más dificil equilibrar el trabajo y la familia, porque los roles de género tradicionales han persistido. Una encuesta de 1997 realizada en las regiones Sureste y Noreste -las cuales representan alrededor de dos terceras partes de la población total del país- encontró que del total de la población trabajadora, $79 \%$ de las mujeres y sólo $29 \%$ de los hombres dedica tiempo al trabajo de la casa. Entre aquellos hombres y mujeres que desarrollan el "trabajo productivo" y el trabajo de la casa, las mujeres dedicaron 61 horas a ambas actividades cada semana, mientras los hombres emplearon solamente 46 horas (Goldani, 1999-2000).

Por consiguiente, la reorganización de la vida familiar en Brasil se ha vuelto cada vez más difícil para las mujeres, y al mismo tiempo

21 La información de la misma encuesta muestra que aunque sólo algunos de los esposos cuidan a los hijos mientras las mujeres trabajan, muchos de ellos deciden qué hacer con los salarios de sus esposas. Del total de mujeres brasileñas en unión que trabajan y tienen ingresos, $63 \%$ decide sobre lo que se hace con su salario, $30 \%$ dice que lo decide con su marido y $7 \%$ declara que su marido decide el uso que se le da a su salario (Goldani, 1999-2000). 
los costos de la crianza de los hijos han aumentado. Criar a los hijos debería ser cada vez más una responsabilidad colectiva y el Estado debería ofrecer programas o planes que permitieran conciliar la vida familiar y el trabajo. Las políticas públicas son cruciales para transformar las responsabilidades de género y en consecuencia la anticoncepción y la crianza de los hijos, como lo han mostrado las experiencias de algunos países industrializados (Corrêa, 2002; Goldani, 2001).

La equidad de género -la "condición posibilitadora" más importante que está en el centro de los enfoques actuales de las políticas de población- es también la llave para evitar una fecundidad con niveles por debajo del reemplazo. Las mujeres de los países industrializados normalmente quieren dos hijos. Si la organización de la sociedad de la "economía del cuidado" no pusiera una enorme carga en los hombros de aquellos que quieren combinar el tener hijos y una carrera, podríamos seguramente observar un incremento en la fecundidad (Barroso, 2001).

\section{Comentarios finales}

A manera de conclusión diría que en Brasil, como en casi todos los demás países, no hay una precondición única o determinante de las elecciones individuales o sociales con relación al comportamiento reproductivo futuro. Sin embargo, el fracaso para identificar los "factores clave" no debería echar para atrás los esfuerzos por incorporar algunos de los elementos mencionados en las proyecciones. Por tanto, entre los indicadores que deben considerarse están: $a$ ) los cambios estructurales que modifican los beneficios y costos tanto de la crianza como de la educación de los hijos; $b$ ) los descensos en la mortalidad infantil y en la mortalidad de adultos jóvenes; $c$ ) el aumento en la escolaridad de las mujeres y en su participación en el mercado de trabajo; $d$ ) el empoderamiento social de las mujeres y una mayor preocupación por la equidad de género; $e$ ) el cambio en las normas sociales en el contexto de un alto valor de los hijos; $f$ ) la planificación familiar como una política pública; $g$ ) la ley de la esterilización; $h$ ) la mezcla de métodos anticonceptivos, e $i$ ) el tamaño de familia deseado o preferido.

Las proyecciones de población muestran las posibles tendencias futuras y ayudan a expandir el horizonte de los hacedores de política más allá de las prioridades a corto plazo. Es importante destacar que la División de Población de las Naciones Unidas publica no sólo una, 
sino tres variantes, todas ellas oficialmente señaladas como igualmente plausibles. La diferencia entre la variante baja y la media para Brasil es de alrededor de 51 millones de personas en 2050, y la diferencia entre la variante media de Naciones Unidas y la proyección de la Oficina del Censo de Estados Unidos es de 40 millones. Tales discrepancias nos llevan a reconocer que las proyecciones sólo son útiles para ubicar los retos que imponen los cambios en las estructuras por sexo y edad; en lugar de negarlas o ignorarlas, tenemos que discutirlas a fin de avanzar en los temas de política pública que tienen que ver con las implicaciones de las tendencias de la población en Brasil, tales como: a) el número creciente de personas adultas mayores y las consecuencias para las relaciones de género e intergeneracionales; $b$ ) las demandas en materia de educación; c) los sistemas de pensiones; $d$ ) las relaciones trabajo-familia, y $e$ ) la equidad de género. Finalmente debemos recordar que las proyecciones cambian cuando la realidad cambia, respondiendo a distintas circunstancias, y esto fortalece la importancia de combinar las evaluaciones erróneas subjetivas, y las probabilísticas basadas en información, para así proporcionar a los usuarios una evaluación más realista de la incertidumbre de los pronósticos demográficos en este nuevo siglo.

\section{Bibliografia}

Alho, Juha M. (1997), "Scenarios, Uncertainty and Conditional Forecasts of the World Population", Journal of the Royal Statistical Society, vol. 160, núm. 1, pp. 71-85.

Barroso, Carmen (2001), "Forum. Is There a Population Implosion?", ECSP Report, núm. 7, Environmental Change and Security Project (ECSP), Woodrow Wilson Center.

Beltrão, Kaizo y Ana Amélia Camarano (2001), "Projeções de população para o Brasil: estimativas de mortalidade", Río de Janeiro, ENCE/ IPEA (mimeo.).

Bemfam (Sociedade Civil Bem-Estar Familiar no Brasil) (1997), Pesquisa Nacional Sobre Demografia e Saude 1996, Río de Janeiro, Bemfam/DHS/IBGE/Unicef/Funap/AID/INAN.

Bercovich, Alicia y Felicia Madeira (1999/2000), "Demographic Discontinuities in Brazil and in the State of Sāo Paulo", Brazilian Joumal of Population Studies, vol. 2, pp. 5-26.

Berquó, Elza (1999), "Ainda a Questão da Esterilização Feminina no Brasil", en Karen Giffin y Sarah H. Costa (coords.), Questöes da Saúde Reprodutiva, Río de Janeiro, Fiocruz. 
(1999a), "Sterilization and Race in São Paulo", en Rebecca Reichmann (comp.), Race in Contemporary Brazil: From Indifference to Equality, University Park, Pensilvania, The Pennsylvania State University Press.

— y Suzana M. Cavenaghi (2002), "The Impacts of the Voluntary Sterilization Law on Reproductive Rights of Men and Women", trabajo presentado en el seminario Population Association of America, Atlanta, Georgia, mayo 9 a 11 (mimeo.).

Caetano, André Junqueira (2001), "Fertility Transition and the Diffusion of Female Sterilization in Northeastern Brazil. The Roles of Medicine and Politics", en el CD-ROM XXIV General Population Conference of International Union for Scientific Studies of Population, Salvador, Bahía, IUSSP.

Camarano, Ana Amélia (1996), Fertility Transition in Brazil in the Twentieth Century: A Comparative Study of Three Areas, tesis de doctorado, London School of Economics and Political Science, Universidad de Londres.

— Herton E. Araujo e Isabella G. Carneiro (1999), "Tendencias da fecundidade brasileira no século XX: Uma visão regional", en Karen Giffin y Sarah H. Costa (coords.), Questöes da saude reprodutiva, Río de Janeiro, Fiocruz.

Carvalho, José Alberto Magno de (1997/1998), "Demographic Dynamics in Brazil Recent Trends and Perspectives", Brazilian Journal of Population Studies, vol. 1, pp. 5-24.

- Claudia J. G. Horta y Simone Wajnman (2000), "Construindo uma medida do tempo medio despendido pelas cortes de mulheres com os cuidadosde filhos pequenos", en XII Encontro Nacional de Estudos Populacionais da ABEP, Caxambu, Minas Gerais.

Corrêa, Sônia (2001), "Is There a Population Implosion?", ECSP Report, vol. 7.

—- Sérgio Piola y Margareth Arilha (1998), "Cairo em Açāo: Estudo de caso Brasil", Parte I: "Perfil, antecedentes, cenário nacional", Brasilia (inédito).

Faria, Vilmar Evangelista (1997/1998), "Government Policy and Fertility Regulations: Unintended Consequences and Perverse Effects", Brazilian Journal of Population Studies, vol. 1.

Formiga Filho, José Ferreira (1999), "Politicas de saúde reprodutiva no Brasil: uma análise do PAISM", en Loren Galvão y Juan Díaz (coords.), Saúde Sexual e Reprodutiva no Brasil: Dilemas e Desafios, cap. 4, São Paulo, Hucitec/Population Council, pp. 151-162.

Goldani, Ana María (2001), "Rethinking Brazilian Fertility Decline", en el CDROM XXIV General Population Conference of International Union for Scientific Studies of Population, Salvador, Bahía, IUSSP.

- (1999/2000), "Gender Relations and Fertility in Northeastern Brazil", Brazilian Journal of Population Studies, ABEP, vol. 2, pp. 69-96.

Hakkert, Ralph (2001), "Levels and Determinants of Wanted and Unwanted Fertility in Latin America", en el CD-ROM XXIV General Population Conference of International Union for Scientific Studies of Poprulation, Salvador, Bahia, IUSSP. 
Horta, Claudia J. Guimarães, José Alberto M. de Carvalho y Luís Armando de M. Frias (2000), "Recomposiçāo da fecundidade por geração para Brasil e regiões: atualização e revisão", trabajo presentado en el XII Encontro Nacional de Estudos Populacionais da ABEP, Caxambu, Minas Gerais (mimeo.).

IBGE (Instituto Brasileiro de Geografia e Estatistica) (2000), Projeção de população do Brasil por sexo e idade para o periodo 1980-2050 (revisão 2000), Projeto UNFPA/Brasil (Bra98/P08), Sistema Integrado de Projeções e Estimativas Populacionais e Indicadores Sócio Demográfico, Departamento de População e Indicadores Sociais.

- (2002), Tábua de Vida, en www.ibge.gov.br/ibge.../tabuadevida/evolucao_da_mortalidade.shtm [acceso en febrero de 2002].

Lam, David y S. Duryea (1999), "Effects of Schooling on Fertility, Labor Supply and Investments in Children, with Evidence from Brazil", Journal of Human Resources, vol. 34, pp. 443-454.

Lazo, Aída Verdugo (1994), "Marital Fertility in Brazil: Differentials by Type of Union and its Importance in the Fertility Transition 1976-1991", DHS Working Paper, núm. 15, Calverton, Maryland.

Leone, Tiziana y André Caetano (2002), "Can the Level of Brazilian Fertility Be Even Lower?", trabajo presentado en el seminario de la Population Association of America, Atlanta, Georgia, mayo 9 a 11.

Lutz, Wolfgang, Warren Sanderson y Sergei Scherbov (1998), "Expert Based Probabilistic Projections", en Lutz Wolfgang, J. Vaupel y D. Ahlburg (comps.), "Frontiers of Population Forecasting", suplemento del Population and Development Review, vol. 24, pp. 139-155.

Martine, George (1996), "Brazil's Fertility Decline, 1965-95: A Fresh Look at Key Factors", Population Development Review, vol. 22, núm. 1, pp. 47-75.

Ministério da Saúde (1997), Brazilian Government, en http:/www.Saude.gov.br [acceso en 2002].

Oliveira, Maria Coleta, Elizabeth D. Bilac y Malvina Muszkat (2001), "Men and Contraception: A Study on Middle-Class Brazilian Men", en el CDROM XXIV General Population Conference of International Union for Scientific Studies of Population, Salvador, Bahía, IUSSP.

Pastore, José y Nelson do Valle Silva (2000), Mobilidade social no Brasil, São Paulo, Makron.

Potter, Joseph E. (1999), "The Persistence of Outmoded Contraceptive Regimes. The Cases of Mexico and Brazil", Population Development Review, vol. 25, núm. 4, pp. 703-739.

—, Carl Schmertmann y Suzana M. Cavenaghi (2002), "Fertility and Development: Evidence from Brazil", Austin, Population Research Center, Universidad de Texas (inédito).

Presser, Harriet B. (1995), "Are the Interests of Women Inherently at Odds with the Interests of Children or the Family? A View Point", en K. Mason y A. Jensen (comps.), Gender and Family Change in Industrialized Countries, Oxford University Press, pp. 279-319. 
Rios Neto, Eduardo G. (2000), "Passado, presente e futuro da fecundidade brasileira", trabajo presentado en el XrV Encontro Nacional de Estudos Populacionais, Brazilian Population Association, Caxambu, Minas Gerais.

Rodríguez Wong, Laura (2001), "Below Replacement Fertility Levels in the Brazilian Metropolitan Cities, 1991-2010", cartel presentado en la Population Association of America, Washington.

Sarti, Cynthia Andersen, C. Simonette y Crístina Bruschini (1990), Gênero em Gerasaão de Renda, São Paulo, Unicef/Fenape.

U.S. Bureau of the Census Bureau (2000), National Poprulation Projections, en http://www.census.gov/population/www/projections/natproj.html [acceso en enero de 2002].

- (1999), World Population Profile: 1998, Washington, US Government Printing Office (Report WP/98).

United Nations (2001), World Population Prospects. The 2000 Revision, vol. I, Comprehensive Tables, y vol. II, The Sex and Age Distribution of Populations, Nueva York, United Nations Publications. 